\title{
BMJ Open Efficacy of adjunctive azithromycin versus single-dose cephalosporin prophylaxis for caesarean scar defect: study protocol for a randomised controlled trial
}

\author{
Yanqing Cai (D) , ${ }^{1}$ Hongjie Pan, ${ }^{1,2}$ Jian Zhang, ${ }^{1}$ Weiwei Cheng, ${ }^{1}$ Yiru Shi, ${ }^{1}$ \\ Min Zeng, ${ }^{3}$ Liye Shi, ${ }^{3}$ Jin Yu, ${ }^{1}$ Ying Shen, ${ }^{1}$ Shan Chen, ${ }^{1}$ Qian Zhu, ${ }^{1}$ Ben W Mol, ${ }^{4,5}$ \\ Ding Huang ${ }^{1}$
}

To cite: Cai Y, Pan H, Zhang J, et al. Efficacy of adjunctive azithromycin versus single-dose cephalosporin prophylaxis for caesarean scar defect: study protocol for a randomised controlled trial. BMJ Open 2020;10:e032379. doi:10.1136/ bmjopen-2019-032379

- Prepublication history for this paper is available online. To view these files, please visit the journal online (http://dx.doi. org/10.1136/bmjopen-2019032379).

Received 15 June 2019 Revised 21 November 2019 Accepted 22 November 2019

D) Check for updates

(c) Author(s) (or their employer(s)) 2020. Re-use permitted under CC BY-NC. No commercial re-use. See rights and permissions. Published by BMJ.

For numbered affiliations see end of article.

Correspondence to

Dr Ding Huang;

dingding123hos@163.com

\section{ABSTRACT}

Introduction Perioperative infections may be considered predictors of caesarean scar defect (CSD), and multidose antibiotics have a protective effect against CSD. However, the ability of adjunctive azithromycin combined with cephalosporin to reduce the prevalence of CSD remains unclear. The planned study aims to clarify the protective effect of antibiotics against CSD and to assess the effectiveness of adjunctive azithromycin prophylaxis for CSD.

Methods and analysis This study is a double-blind, parallel-control randomised clinical trial that will be carried out at the International Peace Maternity and Child Health Hospital. A total of 220 eligible patients will be randomised $(1: 1)$ to receive either adjunctive azithromycin or single-dose cephalosporin $30 \mathrm{~min}$ before the incision. The evaluation criteria are the prevalence and characteristics of CSD as assessed by transvaginal ultrasound (TVU) and saline infusion sonohysterography (SIS) at 42 days, 6 months and 12 months after delivery. The primary outcome will be the prevalence of CSD, and the characteristics of CSD will be assessed by TVU and SIS 42 days after delivery; all other outcomes are secondary.

Ethics and dissemination This protocol received authorisation from the Medical Research Ethics Committee of International Peace Maternity and Child Health Hospital on 25 April 2018 (approval no. GKLW2017-84). The findings will be reported in peer-reviewed publications and presentations at international scientific meetings. Trial registration number ChiCTR-INR-17013272.

\section{INTRODUCTION}

The rate of caesarean section (CS) delivery is increasing worldwide, especially in China due to the prevalence of maternally requested caesarean deliveries and the 30-year national one-child policy. ${ }^{1}$ According to the data from official figures of the China National Bureau of Statistics, there were approximately 17 million newborns in China in 2017, with a caesarean delivery rate of approximately
Strengths and limitations of this study

- This is the first interventional randomised controlled trial with the primary aims of assessing the protective effects of antibiotics against caesarean scar defect (CSD) and investigating the relationship between CSD occurrence and preoperative infection.

- This study is based on our previous cohort study, and we want to study the effects of infection and antibiotics on CSD directly.

- The study is a double-blinded randomised controlled trial with a relatively large sample size and a long follow-up period. In this set, we can find the optimal time point for the early detection of CSD.

- The trial is based in a single centre, which might limit the generalisability of the findings.

- This study is not a randomised controlled trial of different surgical techniques for uterine incision closure, which may be as or more important than antibiotics.

$40 \%$, which means that there are approximately 7 million CS deliveries each year.

There is no universally accepted definition or criterion for caesarean scar defect (CSD). In most studies, CSD is defined as "the thinning of the myometrium or a triangular defect in the myometrium that is contiguous with the endometrial cavity'. ${ }^{2}$ It was stated in the research by Di Spiezio Sardo et $a \hat{l}$ that remaining myometrial thickness (RMT) is defined as 'the distance from the delineation of the endometrium to the serosal surface at the level of the caesarean scar, and total myometrial thickness was measured at the myometrium adjacent to the scar', which refers to adjacent myometrium thickness $(\mathrm{AMT})$. Some researchers have also indicated that a niche should be described as 
the indentation of myometrium with a depth of at least $2 \mathrm{~mm} .{ }^{45}$ Additionally, according to Osser et al, ${ }^{6}$ a large CSD was defined as 'thickness of the remaining myometrium over the defect $\leq 2.2 \mathrm{~mm}$ by TVU and $\leq 2.5 \mathrm{~mm}$ by SIS, ratio between the thickness of the remaining myometrium over the defect and the myometrial thickness adjacent to the defect $\leq 23 \%$ by TVU and $\leq 29 \%$ by SIS for women who had undergone only one caesarean section'. Small CSDs may be definitely common and not considered significant. Nevertheless, large CSDs may lead to some long-term potential sequelae. ${ }^{7}$ In our study, we will focus on the differences in the prevalence rates and outcomes of CSDs and large CSDs. Data on measured parameters such as the height, length, width, RMT, AMT and niche volume of the CSD will be collected to present the characteristics of CSD.

In a random population of women with a history of CS, the prevalence of CSD ranges from $56 \%$ to $84 \%$ and $24 \%$ to $70 \%$ when assessed by transvaginal ultrasound (TVU) with and without contrast enhancement, respectively. ${ }^{8}$ Saline infusion sonohysterography (SIS) is better than traditional TVU for characterising CSD and has a higher sensitivity. ${ }^{9}$ Moreover, sonohysterography can more precisely evaluate the size and shape of the defect and thus the severity. ${ }^{10}$ In this study, we will apply and compare the differences between TVU and SIS.

CSD is associated with abnormal uterine bleeding ( $75 \%$ to $82 \%$ ), postmenstrual spotting (29\% to $34 \%$ ), caesarean scar ectopic pregnancies (1:1800 to $1: 2216)$ and infertility $(32 / 92) .{ }^{41112}$ Uterine dehiscence, uterine rupture, caesarean scar pregnancy and morbidly adherent placenta are also associated with CSD.${ }^{13-15}$ Annually, there are thousands of fatalities and near-death cases caused by uterine rupture or haemorrhage from the CSD. ${ }^{16}$

Our previously published data first revealed a CSD prevalence of $43.4 \%$ (95\% CI $39.1 \%$ to $47.7 \%, \mathrm{n}=514$ ) in Shanghai, which is a cause for concern. ${ }^{17}$ Our cohort study also showed that infection might be an important risk factor for CSD and that multidose antibiotic administration has a significant protective effect compared with single-dose antibiotic administration (CSD prevalence $31.1 \%, 95 \%$ CI $23.8 \%$ to $38.3 \%$ vs $49.0 \%, 95 \%$ CI $43.8 \%$ to $54.3 \%$; aOR $=0.4,95 \%$ CI 0.3 to 0.7$).{ }^{18-20}$ Furthermore, the single-dose cefuroxime subgroup $(\mathrm{n}=190)$ was found to have a significantly lower CSD prevalence than the singledose cefradine subgroup ( $\mathrm{n}=162)(38.9 \%, 95 \%$ CI $34.7 \%$ to $48.6 \%$ vs $56.8 \%, 95 \%$ CI $51.3 \%$ to $66.7 \%$; aOR $=0.5$, $95 \%$ CI 0.3 to $0.8, \mathrm{p}=0.006$ ). These results strongly indicate that antibiotics may be a protective factor for CSD.

According to the results of a study involving pathogen monitoring for nosocomial infections, Ureaplasma urealyticum, Escherichia coli, Enterococcus and Streptococcus are the most common infectious agents. ${ }^{21}{ }^{22}$ Second-generation cephalosporins have a stronger antibacterial effect against E. coli and Enterobacteriaceae than first-generation cephalosporin, while azithromycin has stronger antibacterial and bacteriostatic effects against atypical pathogens, such as Mycoplasma chlamydia and anaerobic bacteria. ${ }^{23}{ }^{24}$ Current recommendations for antibiotic prophylaxis in caesarean delivery include the standard administration of a broad-spectrum antibiotic, most commonly a first-generation cephalosporin, before the skin incision. ${ }^{25}$ Recent reports on adjunctive azithromycin prophylaxis for caesarean delivery showed a significant protective effect against maternal endometritis $(3.8 \%$ vs $6.1 \%, \mathrm{p}=0.02)$, wound infection $(2.4 \%$ vs $6.6 \%, \mathrm{p}<0.001)$ and serious maternal adverse events $(1.5 \%$ vs $2.9 \%, \mathrm{p}=0.03)$ but no significant betweengroup differences in secondary neonatal composite outcomes. ${ }^{26}$ In other words, the addition of azithromycin to caesarean delivery prophylaxis is less costly and leads to better maternal outcomes in index and subsequent deliveries. ${ }^{27-30}$

Therefore, we designed a prospective, randomised, double-blind, parallel-group study to determine whether the prophylactic use of cephalosporin plus azithromycin combination is superior to that of a single dose of cephalosporin in reducing the prevalence of CSD. Our study has a strong theoretical basis and foundation, and this trial may provide evidence of the effective use of antibiotics in clinical practice and data indicating a reduction in the rate of CSD.

\section{Objective and hypothesis}

The objective of this trial is to clarify the protective effects of antibiotics against CSD and to assess the effectiveness of adjunctive azithromycin prophylaxis for CSD. Our hypothesis is that the prophylactic use of cephalosporins combined with azithromycin is superior to a single dose of cephalosporin and can reduce the prevalence of CSD.

\section{METHODS AND ANALYSIS}

\section{Study design and setting}

The study design is that of a double-blind, parallelcontrol randomised clinical trial. Pregnant women with singleton pregnancies in cephalic presentation who have ruptured membranes or signs of labour will be informed about the study and the need to undergo CS by the medical team. The subjects will be randomly divided into two groups. The trial group (group A) will be administered the regular single-dose cefuroxime sodium $(1.5 \mathrm{~g} / 50 \mathrm{~mL} 0.9 \%$ sodium chloride $(\mathrm{NaCl}))$ plus azithromycin $(0.5 \mathrm{~g} / 250 \mathrm{~mL} 0.9 \% \mathrm{NaCl})$. The control group (group B) will be administered the regular single-dose cefuroxime sodium $(1.5 \mathrm{~g} / 50 \mathrm{~mL} 0.9 \% \mathrm{NaCl})$ plus an additional placebo $(250 \mathrm{~mL} 0.9 \% \mathrm{NaCl})$ as an intravenous drip $30 \mathrm{~min}$ before surgery. The prevalence and clinical symptoms of CSD at 6 weeks, 6 months, and 12 months after delivery will be observed with TVU and SIS. We will include 220 women in total. The primary outcome measure will be the prevalence of CSD, and the characteristic of CSD will be assessed by TVU and SIS 42 days after delivery. The secondary outcome measures will be infection indexes and labour results, as shown in figure 1. 


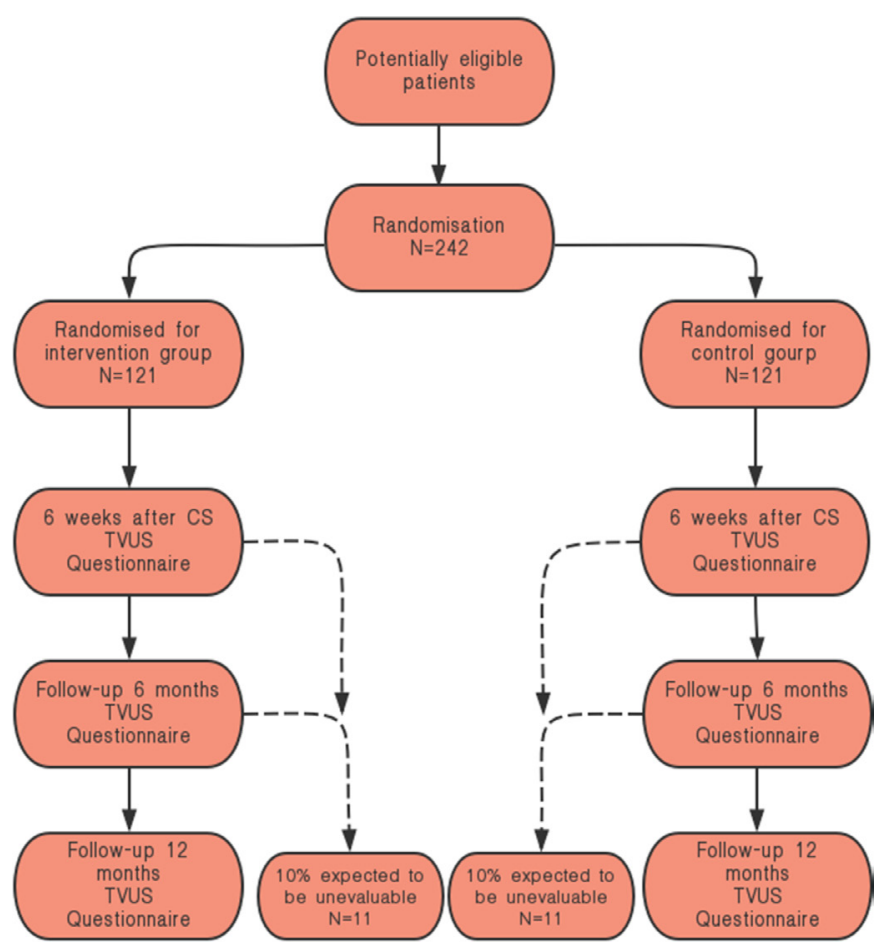

Figure 1 Flowchart of the study. CS, caesarean section; TVUS, transvaginal ultrasonography.

\section{Participants}

Patients will be included in this study if they meet all the following criteria:

1. Han Chinese ethnicity with a maternal age of 18 years or older,

2. Singleton pregnancy with a gestational age $\geq 37$ weeks,

3. Fibrinogen $\geq 2 \mathrm{~g} / \mathrm{L}$, platelet count $\geq 100 \times 10^{9}$ and haemoglobin $\geq 90 \mathrm{~g} / \mathrm{L}$ before surgery,

4. Non-elective caesarean delivery during labour or after membrane rupture and

5. Labour defined as regular contractions with cervical dilation of $3 \mathrm{~cm}$ or with documented cervical changes of at least $1 \mathrm{~cm}$ of dilation or at least $50 \%$ effacement (women with membrane rupture for at least 4 hours will be eligible, regardless of whether labour has started).

Patients will be excluded from participating in the study if one of the following criteria is met:

1. The patient declines to participate in the trial,

2. The patient is known to be allergic to cefuroxime sodium or azithromycin (including an allergy to any kind of macrolide or cephalosporin),

3. The patient was administered azithromycin within 7 days before randomisation,

4. The patient is positive for Group B Streptococcus haemolyticus on screening at approximately 36 weeks.

5. The patient has a diagnosis of a non-reproductive tract infection (appendicitis, upper respiratory infection, urinary tract infection) and needs additional antibiotic treatment,

6. The patient has a severe maternal disease (severe liver or renal dysfunction, pulmonary oedema, cardiac structural abnormality or a condition requiring antiarrhythmic drug use, systematic lupus erythaematosus or inadequately controlled diabetes).

7. The patient has a stillbirth

8. The patient has a preoperative diagnosis of uterine abnormalities (such as uterine malformation, adenomyosis or myoma of the uterus) or

9. The patient has undergone a previous CS.

\section{Randomisation}

Demographic, medical and obstetrical information will be collected at baseline. The patient will then be randomised between the trial group (group A) and the control group (group B) according to her serial number (number 1 to 250 , depending on the time of trial entry). The randomisation codes will be generated by the block of the statistical software at a 1:1 ratio (SPSS, V.22.0, IBM, Armonk, New York, USA). We will generate a list of 250 randomisation codes (A or B). Each code will correspond to one serial number, and each code will be placed in a black envelope with the serial number printed on the outside. Anyone who will have contact with the patient or other researchers will be blinded to the list. Antibiotic prophylaxis will be administered within $30 \mathrm{~min}$ before incision. The medicine will be dissolved in the hospital dispensary, where the patient's envelope will be opened by an independent pharmacist, and the containers will be identical so that no one else can identify the set. The opened envelope will be locked in a non-transparent box until the trial is finished. These settings will ensure that both the participants and investigators or surgeons are blinded to the treatment.

\section{Intervention}

All participants will undergo a standard CS with a lower segment uterine incision and double-layer suturing of the uterine wound. The first layer will be the decidua and total myometrium, and the second layer will be the serosa and the upper half layer of myometrium. All sutures will be continuously unlocked with absorbable suture material. We have three experienced surgeons with the same technical standards who will be responsible for the trial. Each of them will perform approximately $70 \mathrm{CSs}$ randomly in the trial. All the participants will be cared for in the wards, and additional antibiotics will be given if there is a confirmed infection before the patient checks out.

\section{Withdrawal of participants}

The participants will be able to leave the study at any time for any reason if they wish to do so, without any consequences. The investigator will be able to decide to withdraw a subject from the study for urgent medical reasons. After randomisation, if a woman wishes to change her assigned protocol, she will be considered a crossover subject. All subjects will remain in the study for analysis based on the intention-to-treat principle. 
Table 1 Patient's characteristics and data collection

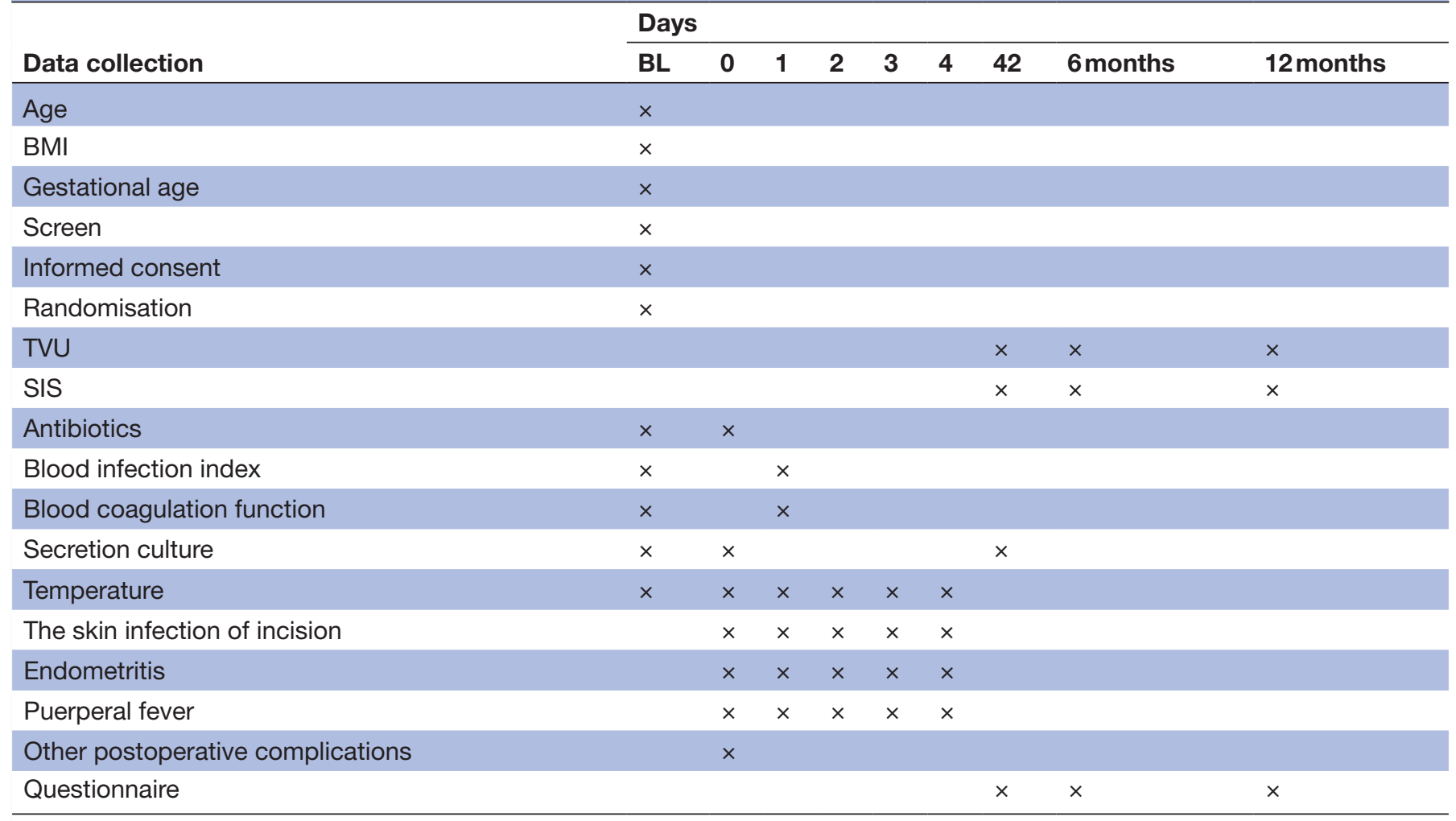

BL, baseline; BMI, body mass index; TVU, transvaginal ultrasound; SIS, saline infusion sonohysterography.

\section{Participant timeline}

The schedules for enrolment, interventions and assessments are summarised in table 1.

The CS will be performed on day 0 , and antibiotic treatment, which will be randomly assigned, will be started on day 1.

All included patients will undergo randomisation and treatment. We will conduct preoperative and postoperative routine blood tests and detect C-reactive protein (CRP) levels, procalcitonin (PCT) levels and blood coagulation function. We will also culture vaginal secretions before surgery and at 42 days postpartum.

Clinical evaluations of the efficacy and safety of antiinfection prophylaxis will be performed during hospitalisation and will include evaluations of the incidence of puerperal fever, skin infections at the incision site, endometritis and other postoperative complications.

Clinical symptoms as well as the prevalence and characteristics of CSD at 6 weeks, 6 months and 12 months after caesarean delivery will be documented.

\section{Primary outcome measure}

The prevalence of CSD, and the characteristic of CSD will be assessed by TVU and SIS 42 days after delivery.

\section{Secondary outcome measure}

1. The changes in infection indexes, such as routine blood examinations including CRP and PCT before and after surgery.
2. Presurgical vaginal secretion culture results and intraoperative uterine cavity culture results.

3. Body temperature 6 hours before and 72 hours after CS.

4. Postoperative morbidity, endometritis and incisional infection after surgery and severe infection 42 days after delivery.

5. Type and dosage of all antibiotics administered postoperatively according to standard processes.

6. Responses to postpartum questionnaires at approximately 6 weeks, 6 months and 12 months.

7. Follow-up data from TVU and SIS evaluations performed at 6 months and 12 months after delivery.

8. The size and position of the uterus and the height, length, width, RMT, AMT and niche volume of the defects.

\section{STATISTICAL CONSIDERATIONS \\ Sample size calculation}

Based on the findings of previous studies, we concluded that the occurrence of CSD decreases from $47.9 \%$ to $29 \%$ after the administration of multidose antibiotics during emergency caesarean delivery. We will predict the occurrence of CSD after using two prophylactic antibiotics with PASS sample prediction software V.11.0. We will need to include 220 women in total (two groups of 110 women), with an alpha error of 0.05 . Assuming a $10 \%$ dropout rate, we will need to randomise a total of 242 participants (121 participants per group). 


\section{Statistical analysis}

For analysis, the adopted Electronic Data Capture ( EDC ) c system will be used to collect data uniformly and record all relevant personal information for data collection points. The method of automatic computer verification, which will include data that clearly do not conform to the conventional standards and among which values are missing, will be adopted. Researchers will be required to check the original data, and all changes in the data will be managed with traces. Query tables will be checked if necessary.

For the analysis of the data, t-tests, analysis of variance, $\chi^{2}$ tests and other differential tests will be carried out with SPSS software.

Descriptive statistics will be used to analyse demographic data and laboratory indicators, such as basic information from the preoperative period, from the postoperative hospitalisation period and at 6 weeks postsurgery. Preoperative and postoperative indicators will be used for baseline data, and the normality of the distribution of continuous variables from the baseline data will be tested. Normally distributed data will be reported as the mean and SD, and the two groups will be assessed by the independent samples t-test; if the data are non-normally distributed, a non-parametric analysis will be performed, and the data will be reported as the median and IQR. We will use the $\chi^{2}$ test or Fisher's exact test to analyse categorical variables.

We will apply the $\chi^{2}$ test to assess the primary outcome to compare the occurrence rate of the CSD in two groups.

We will apply the $\mathrm{t}$-test, analysis of variance and $\chi^{2}$ test to evaluate the secondary outcome according to the characteristics of the endpoint.

\section{Patient and public involvement}

Neither the patients nor the public will be involved in the study design. They will also not be involved in the recruitment process or conduct of the study. The results will be disseminated to patients via an open access publication and our local trials teams.

\section{DISCUSSION}

Since the mechanism of wound healing in uterine caesarean incisions is not fully known and since the natural development of CSD over time is still unclear, uterine scars and CSD may change over time. One prospective observational study showed that CSD occurred from 6 weeks to 6 months or longer after surgery. ${ }^{30}$ Another prospective study reported that the statuses of some women with or without CSD showed a reversal between 6 weeks and 6 months after surgery $(90.7 \%$ at 6 weeks and $93.1 \%$ at 6 months (OR 0.96 (0.61 to 1.49$), \mathrm{p}=0.91)$ ). ${ }^{31}$ This trial is based on our previous cohort study in which CSD was observed at 6 weeks postpartum. We will set the initial CSD assessment time point as 6 weeks postpartum and follow patients for 1 year, aiming to find the optimal time point for the early detection of CSD. Our sample size calculation is also based on previously published data, which were based on the measurement of CSD with TVU and may have resulted in an underestimation. ${ }^{16}$ Since we believe that the difference between the two groups will be more significant when CSD is measured with SIS, the sample size could be large enough to observe a difference. We believe that surgical techniques of uterine incision and closure may be important in the occurrence of CSD, and there are many trials that are ongoing or that already have results. ${ }^{32}{ }^{33}$ We will focus on the effects of infection and antibiotics against CSD based on the strong indication of our previous study.

\section{Author affiliations}

${ }^{1}$ Obstetrics and Gynecology, International Peace Maternity and Child Health Hospital, Shanghai, China

${ }^{2}$ Obstetrics and Gynecology, Zhejiang University School of Medicine Sir Run Run Shaw Hospital, Hangzhou, China

${ }^{3}$ Ultrasound, International Peace Maternity and Child Health Hospital, Shanghai, China

${ }^{4}$ Obstetrics and Gynecology, Monash University, Melbourne, Victoria, Australia ${ }^{5}$ Robinson Research Institute, School of Medicine, The University of Adelaide, Adelaide, South Australia, Australia

Acknowledgements The authors acknowledge Yanfei Zhang for dissolving the medicine for free and for his confidentiality regarding all the participants and personnel.

Contributors YC, HP and BWM are responsible for the overall logistical aspects of the trial and the drafting of this paper. DH, BWM, WC and JZ designed the trial and were responsible for the development of the protocol. The senior doctors $\mathrm{DH}$ and YS are the main operators of the randomised controlled trial. MZ and LS, senior ultrasound doctors, are responsible for the ultrasonographic measurements of the uterus diverticulum. Jin Yu is responsible for the design of the randomisation codes and production of the black envelopes. YS, SC, QZ is contributing to the protocol by carrying out literature retrieval, specimen collection, data analysis and follow-up visits. $\mathrm{DH}$ is responsible for the project and is contributing to the organisation and operation of the trial.

Funding This trial received funding from the Shanghai Shenkang Hospital Development Center Clinical Science and Technology Innovation Project (item number SHDC12017X05) and it was supported by the medical engineering cross youth funds from Shanghai Jiao Tong University (item number YG2017QN38).

\section{Competing interests None declared.}

Patient consent for publication Parental/guardian consent obtained.

Ethics approval The study was approved by the Medical Research Ethics Committee of International Peace Maternity and Child Health Hospital (approval no. GKLW2017- 84).

Provenance and peer review Not commissioned; externally peer reviewed.

Open access This is an open access article distributed in accordance with the Creative Commons Attribution Non Commercial (CC BY-NC 4.0) license, which permits others to distribute, remix, adapt, build upon this work non-commercially, and license their derivative works on different terms, provided the original work is properly cited, appropriate credit is given, any changes made indicated, and the use is non-commercial. See: http://creativecommons.org/licenses/by-nc/4.0/.

ORCID iD

Yanqing Cai http://orcid.org/0000-0002-4336-3659

\section{REFERENCES}

1 Betrán AP, Ye J, Moller A-B, et al. The increasing trend in caesarean section rates: global, regional and national estimates: 1990-2014. PLoS One 2016;11:e0148343.

2 Tower AM, Frishman GN. Cesarean scar defects: an underrecognized cause of abnormal uterine bleeding and other gynecologic complications. J Minim Invasive Gynecol 2013;20:562-72. 
3 Di Spiezio Sardo A, Saccone G, McCurdy R, et al. Risk of Cesarean scar defect following single- $v$ s double-layer uterine closure: systematic review and meta-analysis of randomized controlled trials. Ultrasound Obstet Gynecol 2017:50:578-83.

4 Allornuvor GFN, Xue M, Zhu X, et al. The definition, aetiology, presentation, diagnosis and management of previous caesarean scar defects. J Obstet Gynaecol 2013;33:759-63.

5 Sholapurkar SL. Etiology of cesarean uterine scar defect (niche): detailed critical analysis of hypotheses and prevention strategies and peritoneal closure debate. J Clin Med Res 2018;10:166-73.

6 Vikhareva Osser O, Jokubkiene L, Valentin L. Cesarean section scar defects: agreement between transvaginal sonographic findings with and without saline contrast enhancement. Ultrasound Obstet Gynecol 2010;35:75-83.

7 Yao M, Wang W, Zhou J, et al. Cesarean section scar diverticulum evaluation by saline contrast-enhanced magnetic resonance imaging: the relationship between variable parameters and longer menstrual bleeding. J Obstet Gynaecol Res 2017;43:696-704.

8 Bij de Vaate AJM, van der Voet LF, Naji O, et al. Prevalence, potential risk factors for development and symptoms related to the presence of uterine niches following cesarean section: systematic review. Ultrasound Obstet Gynecol 2014;43:372-82.

9 Nieuwenhuis LL, Hermans FJ, Bij de Vaate AJM, et al. Three-dimensional saline infusion sonography compared to two-dimensional saline infusion sonography for the diagnosis of focal intracavitary lesions. Cochrane Database Syst Rev 2017;5:CD011126.

10 Baranov A, Gunnarsson G, Salvesen K. Å., et al. Assessment of cesarean hysterotomy scar in non-pregnant women: reliability of transvaginal sonography with and without contrast enhancement. Ultrasound Obstet Gynecol 2016;47:499-505.

11 van der Voet LF, Vervoort AJ, Veersema S, et al. Minimally invasive therapy for gynaecological symptoms related to a niche in the caesarean scar: a systematic review. BJOG 2014;121:145-56.

12 Tulandi T, Cohen A. Emerging manifestations of cesarean scar defect in Reproductive-aged women. J Minim Invasive Gynecol 2016;23:893-902.

13 Pomorski M, Fuchs T, Zimmer M. Prediction of uterine dehiscence using ultrasonographic parameters of cesarean section scar in the nonpregnant uterus: a prospective observational study. BMC Pregnancy Childbirth 2014;14:365-71.

14 Timor-Tritsch IE, Monteagudo A, Cali G, et al. Cesarean scar pregnancy is a precursor of morbidly adherent placenta. Ultrasound Obstet Gynecol 2014;44:346-53.

15 Vervoort AJ, Uittenbogaard LB, Hehenkamp WJ, et al. Why do niches develop in caesarean uterine scars? Hypotheses on the aetiology of niche development. Hum Reprod 2015;30:2695-702.

16 Kaelin Agten A, Cali G, Monteagudo A, et al. The clinical outcome of cesarean scar pregnancies implanted "on the scar" versus "in the niche". Am J Obstet Gynecol 2017;216:510.e1-10.

17 Pan $\mathrm{H}$, Zeng $\mathrm{M}, \mathrm{Xu}$ T, et al. The prevalence and risk predictors of cesarean scar defect at 6 weeks postpartum in Shanghai, China: A prospective cohort study. Acta Obstet Gynecol Scand 2019;98:413-22.
18 Smaill FM, Grivell RM. Antibiotic prophylaxis versus no prophylaxis for preventing infection after cesarean section. Cochrane Database Syst Rev 2014;10:CD007482.

19 Gyte GM, Dou L, Vazquez JC. Different classes of antibiotics given to women routinely for preventing infection at caesarean section. Cochrane Database Syst Rev 2014;11:CD008726.

20 Allen J, David M, Veerman JL. Systematic review of the costeffectiveness of preoperative antibiotic prophylaxis in reducing surgical-site infection. BJS Open 2018;2:81-98.

21 D'Angelo LJ, Sokol RJ. Determinants of postpartum morbidity in laboring monitored patients: a reassessment of the bacteriology of the amniotic fluid during labor. Am J Obstet Gynecol 1980;136:575-8.

22 Lamey JR, Eschenbach DA, Mitchell SH, et al. Isolation of mycoplasmas and bacteria from the blood of postpartum women. Am J Obstet Gynecol 1982;143:104-12.

23 Mackeen AD, Packard RE, Ota E, et al. Timing of intravenous prophylactic antibiotics for preventing postpartum infectious morbidity in women undergoing cesarean delivery. Cochrane Database Syst Rev 2014;12:CD009516.

24 Purba AKR, Setiawan D, Bathoorn E, et al. Prevention of surgical site infections: a systematic review of cost analyses in the use of prophylactic antibiotics. Front Pharmacol 2018;9:776.

25 Sun J, Ding M, Liu J, et al. Prophylactic administration of cefazolin prior to skin incision versus antibiotics at cord clamping in preventing postcesarean infectious morbidity: a systematic review and metaanalysis of randomized controlled trials. Gynecol Obstet Invest 2013;75:175-8.

26 Tita ATN, Boggess K, Saade G. Adjunctive azithromycin prophylaxis for cesarean delivery. N Engl J Med 2017;376:182.

27 Ward E, Duff P. A comparison of 3 antibiotic regimens for prevention of postcesarean endometritis: an historical cohort study. Am J Obstet Gynecol 2016;214:751.e1-4.

28 Sutton AL, Acosta EP, Larson KB, et al. Perinatal pharmacokinetics of azithromycin for cesarean prophylaxis. Am J Obstet Gynecol 2015;212:812.e1-6.

29 Skeith AE, Niu B, Valent AM, et al. Adding azithromycin to cephalosporin for cesarean delivery infection prophylaxis: a costeffectiveness analysis. Obstet Gynecol 2017;130:1279-84.

30 Harper LM, Kilgore M, Szychowski JM, et al. Economic evaluation of adjunctive azithromycin prophylaxis for cesarean delivery. Obstet Gynecol 2017;130:328-34.

31 Bamberg C, Hinkson L, Dudenhausen JW, et al. Longitudinal transvaginal ultrasound evaluation of cesarean scar niche incidence and depth in the first two years after single- or double-layer uterotomy closure: a randomized controlled trial. Acta Obstet Gynecol Scand 2017;96:1484-9.

32 Dosedla E, Calda P. Can the final sonographic assessment of the cesarean section scar be predicted 6 weeks after the operation? Taiwan J Obstet Gynecol 2016;55:718-20.

33 Roberge S, Demers S, Berghella V, et al. Impact of single- vs double-layer closure on adverse outcomes and uterine scar defect: a systematic review and metaanalysis. Am J Obstet Gynecol 2014;211:453-60. 